\title{
Modelos Matemáticos para Otimização do Tráfego Urbano Semaforizado*
}

\author{
M. LACORTT ${ }^{1}$, M. KRIPKA ${ }^{2}$ e R.M.L. KRIPKA ${ }^{1 * *}$
}

Recebido em 30 setembro, 2012 / Aceito em 19 setembro, 2013

\begin{abstract}
RESUMO. Apresentam-se dois modelos matemáticos de otimização os quais, considerando os dados do trânsito da região que se pretende otimizar, possibilitam fornecer tempos de verde que visam minimizar o tempo de percurso veicular. Também são apresentados os resultados computacionais obtidos com a investigação desses modelos em um estudo realizado na região central da cidade de Passo Fundo, RS. Os resultados apontam para uma melhora significativa no fluxo veicular, dando respaldo a um possível emprego destes modelos em situação real.
\end{abstract}

Palavras-chave: trânsito, otimização combinatória, programação semafórica.

\section{INTRODUÇÃO}

O transporte veicular é um artifício indispensável para a mobilidade humana, do qual a maioria da população mundial usufrui para se deslocar de forma rápida e segura, sendo estas características as mais importantes para se classificar um bom meio de transporte. As crescentes facilidades em adquirir um meio de transporte próprio, o desenvolvimento econômico, a má qualidade do transporte público e o aumento populacional constituem-se em fatores decisórios para o acúmulo de veículos nos centros urbanos. Em razão do aumento contínuo da frota veicular, os sistemas de trânsito atuais da maioria das cidades acabam não comportando o grande número de veículos existentes. Por consequência, surgem os inevitáveis congestionamentos e, com esses, vários problemas à sociedade, como alto nível de estresse, poluição, acidentes e prejuízos econômicos, sendo estes últimos decorrentes do alto consumo de combustível e desperdício de tempo. Desse modo, o problema referente ao trânsito é uma das principais preocupações dos governantes, dos profissionais da área de planejamento urbano e da população, de forma geral, tendo em vista que todos que usufruem de alguma maneira do sistema viário urbano passam a ser afetados por tal situação.

\footnotetext{
*Trabalho apresentado no Congresso Nacional de Matemática Aplicada e Computacional - CNMAC 2012.

** Autor correspondente: Rosana Maria Luvezute Kripka

${ }^{1}$ Instituto de Ciências Exatas e Geociências, ICEG, UPF - Universidade de Passo Fundo, 99001-970 Passo Fundo, RS, Brasil. E-mails: lacortt@upf.br; rkripka@upf.br

${ }^{2}$ Programa de Pós-Graduação em Engenharia, PPGEng, UPF - Universidade de Passo Fundo, 99001-970 Passo Fundo, RS, Brasil. E-mail: mkripka@upf.br
} 
Atualmente, existem vários dispositivos eletrônicos desenvolvidos com a finalidade de organizar o trânsito para que este flua de forma rápida e segura, dentre os quais se destaca o semáforo, cujo desempenho está diretamente relacionado à sua programação. Visando minimizar os problemas de tráfego nas cidades de médio e grande porte, diversos softwares e algoritmos têm sido desenvolvidos, tais como o sistema Siri ([14]), Atefi ([12]), Scoot e Transyt ([13]), sendo este último o mais citado na literatura. No entanto, esses softwares são de alto custo para aquisição e necessitam de treinamento para sua utilização. Dessa forma, diante da falta de recursos e de mão de obra especializada por parte da maioria das prefeituras brasileiras, os problemas referentes ao trânsito das cidades brasileiras não têm sido comumente estudados com o emprego dos sistemas citados.

Neste sentido, o presente trabalho tem como objetivo investigar e aplicar modelagens matemáticas de otimização para programação de semáforos que, automatizadas, forneçam tempos de verde que reduzam de forma considerável o tempo de percurso veicular, especialmente nas regiões centrais de cidades de médio e grande porte, cujo trânsito é intenso e controlado por semáforos.

O presente trabalho encontra-se dividido como segue: na Seção 2 são apresentados conceitos básicos relacionados à engenharia de tráfego; na Seção 3 são descritos os modelos empregados para a otimização da programação semafórica; na Seção 4 é apresentado um estudo desenvolvido a partir da aplicação dos modelos, bem como os resultados obtidos; por fim, na Seção 5, apresentam-se as conclusões do trabalho.

\section{ENGENHARIA DE TRÁFEGO}

A engenharia de tráfego é um ramo da ciência destinada ao estudo de conceitos pertinentes a elementos dos sistemas de tráfego em geral, contribuindo decisivamente para os planejamentos e tomadas de decisões relacionadas ao trânsito. Diversos conceitos são estudados e definidos, dentre os quais destacam-se: Aproximações: são os trechos de via que convergem para a interseção; Fase: é a aplicação completa de uma sequência de indicação de cores de um semáforo; Estágio ou intervalo: é o período de tempo dentro do ciclo dado a cada uma das cores da sequência e Ciclo: é o tempo total para a completa sequência de sinalização de uma interseção ([2]).

A regulagem de semáforos proporciona o bom desempenho do tráfego em termos de fluidez e segurança e, para realizá-la, é necessário ter conhecimento das principais características do trânsito pertencentes às vias, assim como, conceitos, técnicas e expressões que quantificam tais características. A capacidade de uma aproximação sinalizada é definida como sendo o número máximo de veículos capazes de atravessar uma interseção num dado período de tempo. É determinada pelo fluxo de saturação e pelo tempo de verde do semáforo. Quanto maior o tempo de verde de um semáforo, maior a quantidade de veículos liberados. Já o fluxo de saturação de uma interseção consiste no fluxo que seria obtido se houvesse uma fila de veículos na aproximação e a ela fosse dado $100 \%$ de tempo de verde do cruzamento (ou seja, escoamento ininterrupto). O fluxo de saturação é expresso em unidade de veículos por hora de tempo de verde (veículos/ htv). Cabe destacar que o tempo de verde de uma dada fase não é totalmente aproveitado. No 
início do tempo de verde há uma perda ou atraso inicial, em razão do tempo que o motorista leva para atingir a velocidade normal de operação. Nesta etapa a taxa de escoamento de veículos é baixa. À medida que o tempo passa, essa taxa vai aumentando e, após alguns segundos, atinge um valor máximo, razoavelmente constante, que é o fluxo de saturação. Após isso, durante o término do fluxo de verde e início do amarelo, o fluxo ainda continua máximo por alguns segundos e, então, começa a decair, até atingir o valor zero. Assim, no Manual de Semáforos do DENATRAN ([2]) define-se o tempo de verde efetivo como o período do tempo de verde no qual ocorre o fluxo máximo de veículos (fluxo de saturação). Esse é o tempo realmente aproveitado pela fase à qual foi alocado o tempo de verde na aproximação. Define-se ainda o tempo perdido ou tempo morto como o tempo que se perde em virtude das reações dos motoristas no início e no fim do tempo de verde de uma dada fase e durante o qual não há fluxo.

Para o cálculo dos parâmetros de trânsito descritos nos parágrafos anteriores, bem como de outros parâmetros de desempenho necessários para a regulagem de semáforos isolados, propostos por Webster, ([2], p. 63-67), são utilizadas as equações descritas a seguir com suas respectivas notações.

Para determinar a capacidade de uma aproximação sinalizada $\left(C_{p}\right)$, medida em veículos por hora, é multiplicado o fluxo de saturação $(S)$ pelo tempo de verde efetivo $\left(g_{e f}\right)$, dividido pelo tempo de ciclo $(C)$, ambos em segundos, conforme apresentado na Equação (2.1).

$$
C_{p}=S \cdot\left(\frac{g_{e f}}{C}\right)
$$

Já o tempo de verde efetivo ( $\left.g_{e f}\right)$ é definido pela soma do tempo de verde normal $(g)$ ao tempo de amarelo $\left(t_{a}\right)$, subtraídos do tempo perdido por fase $\left(I_{i}\right)$, podendo ser calculado pela Equação (2.2).

$$
g_{e f}=\left(g+t_{a}\right)-I_{i}
$$

Deve-se assegurar que a duração do período de verde de uma fase não seja inferior ao limite mínimo, estabelecido em 10s. Segundo Leite (citado por [9]), o tempo de amarelo deve ser da ordem de: $3 \mathrm{~s}$, quando a velocidade for menor ou igual a $50 \mathrm{~km} / \mathrm{h} ; 4 \mathrm{~s}$, quando a velocidade for maior que $50 \mathrm{~km} / \mathrm{h}$ e menor ou igual a $80 \mathrm{~km} / \mathrm{h}$ e $5 \mathrm{~s}$, quando a velocidade for maior que $80 \mathrm{~km} / \mathrm{h}$.

O tempo perdido por ciclo $\left(T_{p}\right)$, segundo Lima ([9]), pode ser definido pelo somatório dos tempos perdidos por fase $\left(I_{i}\right)$, mais o somatório dos tempos perdidos menos os tempos de amarelo das fases envolvidas $\left(t_{a}\right)$, dado pela Equação (2.3). Para o mesmo autor, caso não se faça o uso da Equação (2.3), o tempo perdido por fase deve ser de 2 a 3 segundos, ou deve-se usar o tempo perdido total como a soma dos tempos de amarelo.

$$
T_{p}=\sum_{i=1}^{n} I_{i}+\sum_{i=1}^{n}\left(I-t_{a}\right)_{i}
$$

Seguindo com a modelagem de Webster, o fluxo de saturação $(S)$ de uma aproximação, medido em veículos por hora de tempo verde (v/htv), pode ser estimado pela Equação (2.4), em que $L$ corresponde à largura da aproximação, em metros.

$$
S=525 \cdot L
$$


A taxa de ocupação ( $y$ ) de uma dada aproximação (ou corrente de tráfego) é a relação entre a demanda de tráfego ou fluxo veicular $(q)$ e o fluxo de saturação $(S)$ definido em (2.4), e é calculada pela Equação (2.5).

$$
y=\frac{q}{S}
$$

A taxa de ocupação é uma medida absoluta da solicitação de tráfego numa aproximação. Por exemplo: uma taxa de ocupação $y=0,5$ corresponde a dizer que a demanda atual da aproximação é a metade da capacidade potencial máxima de atendimento.

O tempo de verde é alocado de acordo com a solicitação de atendimento das aproximações. Logo, quanto maior for a demanda das aproximações de uma dada fase, maior o tempo de verde a ser alocado para a mesma.

O grau de saturação $(X)$, definido como sendo a relação entre a demanda de tráfego $(q)$ e a capacidade de atendimento de uma aproximação $\left(C_{p}\right)$ definida em (2.1), é calculado pela Equação (2.6).

$$
X=\frac{q}{C_{p}}
$$

O grau de saturação é um coeficiente que indica, dentro das condições reais de operação da interseção, o quanto a demanda está próxima da capacidade horária de escoamento. Quando o grau de saturação for superior a 1, significa que a solicitação é maior que a capacidade de atendimento da aproximação, ou seja, ocorre a formação de fila, o que gera um congestionamento em razão dos veículos que chegam e não são atendidos.

Webster também formulou o cálculo de ciclo ótimo $\left(C_{o}\right)$, obtido através de uma série de estudos e simulações computacionais do comportamento do fluxo de tráfego e da relação entre atraso médio e tamanho de ciclo, para intersecções sinalizadas isoladas, em função apenas do tempo de perda total $\left(T_{p}\right)$ e do somatório das taxas de ocupação críticas $\left(y_{c_{i}}\right)$, conforme a Equação (2.7).

$$
C_{o}=\frac{1,5 \cdot T_{p}+5}{1-\sum_{i=1}^{n} y_{c_{i}}}
$$

Apesar de os modelos de Webster serem, em sua maioria, originados em 1964, têm grande validade na atualidade, visto que muitos softwares de programação de semáforos têm como base seus modelos de programação semafórica.

Outro conceito importante para coordenação de semáforos consiste no atraso veicular, o qual é relativo à parcela do tempo consumido em um deslocamento que excede ao tempo desejado pelo usuário ([15], p. 5), ou seja, devido às paradas que o motorista é obrigado a realizar ao longo do deslocamento e ao fato de trafegar com velocidade menor que a desejada.

Para determinação do atraso médio existem diversas fórmulas, as quais envolvem os parâmetros do trânsito do cruzamento específico, conforme o Manual de semáforos do DENATRAN ([2], p. 73). Por meio de uma série de pesquisas, Webster deduziu a fórmula da Equação (2.8) para o atraso médio total sofrido por um veículo, na qual os dois primeiros termos da equação referem- 
se ao atraso uniforme e aleatório, respectivamente, e o terceiro termo é um fator de correção correspondente a cerca de $5 \%$ a $10 \%$ do atraso total.

$$
d_{m}=\frac{C\left(1-\lambda_{i}\right)^{2}}{2\left(1-X_{i} \lambda_{i}\right)}+\frac{X_{i}^{2}}{2 q_{i}\left(1-X_{i}\right)}-0,65\left(\frac{C}{q_{i}^{2}}\right)^{\frac{1}{3}} X_{i}^{\left(2+5 \lambda_{i}\right)}
$$

em que: $d_{m}$ : Atraso médio total sofrido por um veículo; $C$ : Tempo de ciclo; $\lambda_{i}$ : Relação entre o tempo de verde efetivo e o tempo de ciclo $\left(\frac{g_{e} f_{i}}{C}\right) ; X_{i}$ : Grau de saturação da aproximação e $q_{i}$ : Demanda (veículos/segundos).

O cálculo de atraso médio por veículo também pode ser estimado pela forma reduzida de Webster, através da Equação (2.9).

$$
d_{m r}=\frac{9}{10}\left[\frac{C\left(1-\lambda_{i}\right)^{2}}{2\left(1-X_{i} \lambda_{i}\right)}+\frac{X_{i}^{2}}{2 q_{i}\left(1-X_{i}\right)}\right]
$$

O número de paradas sofridas por veículos em uma aproximação é outro fator de desempenho importante, utilizado neste trabalho. Segundo Chile ([3], p. 111), estima-se, pela Equação (2.10), a proporção percentual de veículos detidos $(h)$, que param na aproximação pelo menos uma vez.

$$
h=\frac{1-\lambda_{i}}{1-y}
$$

Além disso, considerou-se o tempo de deslocamento $\left(T_{d}\right)$ em um conjunto de aproximações o qual, segundo Gobbo ([4], p. 13), é definido pelo produto do fluxo ( $q$ ) pela soma do atraso veicular $\left(A_{v}\right)$ mais comprimento da aproximação $(d)$, dividido pela velocidade esperada de deslocamento do fluxo na aproximação $\left(V_{p}\right)$, conforme a Equação (2.11).

$$
T_{d}=q \cdot\left(\frac{d}{V_{p}}+A_{v}\right)
$$

\section{MODELOS PARA OTIMIZAÇÃO DO TRÁFEGO URBANO SEMAFORIZADO}

Neste trabalho são apresentados dois modelos matemáticos de otimização, os quais foram investigados e aplicados a um estudo de caso real. Estes modelos foram elaborados considerando conceitos e expressões expostos na Seção 2. Com estes modelos, objetiva-se otimizar, através das variáveis tempos de verde, o tempo de percurso veicular em vias com trânsito semaforizado.

\subsection{Modelo 1}

Propõe-se um modelo que visa representar de forma significativa situações reais do cotidiano do tráfego urbano semaforizado, onde foram consideradas as expressões de atraso uniforme e atraso aleatório de Webster ([2]), bem como a expressão para determinação do número de paradas sofridas por um veículo, exposta por Chile ([3]), sendo que no modelo em questão, o tempo de verde considerado não é o efetivo e sim o tempo de verde de operação do semáforo (verde normal). 
Diante destas expressões, obteve-se uma função objetivo que representa o Índice de Desempenho de um veículo que trafegue em uma via semaforizada ( $I D)$, expresso na Equação (3.1) como o somatório dos indicativos de desempenho de cada cruzamento da região analisada.

$$
I D=\sum_{i=1}^{n_{c}}\left(A U_{i}+A L_{i}+K P_{i}\right)
$$

em que: $n_{c}$ : Número de cruzamentos; $A U_{i}$ : Atraso médio uniforme do cruzamento $i ; A L_{i}$ : Atraso médio aleatório do cruzamento $i ; K$ : Fator de penalidade de parada; $P_{i}$ : Número de paradas sofridas por um veículo no cruzamento $i$.

O valor do $K$, segundo Chile ([3]), pode variar de 10 a 60, dependendo da importância imposta ao número de paradas. As composições matemáticas de cada termo estão descritas nas Equações (3.2), (3.3) e (3.4), respectivamente. A expressão que corresponde ao atraso uniforme $(A U)$ sofrido por um veículo é dada pela Equação (3.2).

$$
A U=\frac{C\left(1-\lambda n_{i}\right)^{2}}{2\left(1-X_{i} \lambda n_{i}\right)}
$$

em que: $\lambda n_{i}$ : Relação entre o tempo de verde normal e o tempo de ciclo $\frac{g_{i}}{C}$ e $X_{i}$ : Grau de saturação da aproximação $i$.

Já o cálculo do atraso aleatório $(A L)$ é obtido pela Equação (3.3).

$$
A L=\frac{X_{i}^{2}}{4\left(1-X_{i}\right)}
$$

O cálculo do número de paradas $(P)$ sofridas por um veículo pode ser obtido pela Equação (3.4).

$$
P=\left(\frac{1-\frac{g_{i}}{C}}{1-\frac{q}{S}}\right)
$$

em que: $g_{i}$ : Tempo de verde; $S$ : Fluxo de saturação e $q$ : Fluxo veicular.

As equações, (3.1) a (3.4), simulam, em situações reais, a periodicidade desses fatores de desempenho em $n_{c}$ cruzamentos de uma rede composta por semáforos, o qual, como descrito anteriormente, terá como variáveis os tempos de verde. Com a obtenção dos tempos de verde para cada cruzamento da rede otimizada, as demais temporizações podem ser obtidas facilmente. Os tempos de ciclo, vermelho total e tempos de amarelo são definidos anteriormente à otimização e os demais tempos são obtidos por meio do diagrama de tempos, disponível na literatura (([2]), p. 62). Diagrama de tempos consiste na representação gráfica dos intervalos de tempo do ciclo, que associa os instantes de mudança dos estágios com a sequência de cores e duração dos estados de cada fase.

De forma resumida, pode-se dizer que, no Modelo 1 de otimização proposto, busca-se calcular o tempo de verde ótimo $\left(g_{i}\right)$ da aproximação $i$ que possibilite minimizar o atraso e o número de paradas sofridas por um veículo em uma rede semaforizada. Como dados de entrada tem-se: 
o número de cruzamentos, o fluxo de saturação e fluxo veicular de cada fase desses cruzamentos, o tamanho do ciclo e o tempo perdido. Assim, de forma genérica, o Modelo 1 de otimização pode ser representado da seguinte forma:

$$
\text { Minimizar } \quad I D=\sum_{i=1}^{n_{c}}\left(A U_{i}+A L_{i}+K P_{i}\right)
$$

Sujeito à $\quad T_{p} \leq g_{i} \leq C, i=1, \ldots, n_{c}$

em que: $T_{p}$ : Tempo perdido.

\subsection{Modelo 2}

Assim como o Modelo 1, o Modelo 2 é composto por uma expressão que indica o desempenho de uma via semaforizada, onde a composição da função objetivo tem como base a fórmula do tempo de deslocamento de Gobbo ([4], p. 13). A fórmula proposta diferencia-se por representar o tempo de deslocamento de um veículo, ao passo que a fórmula descrita por Gobbo representa o tempo de todo o fluxo veicular presente na via. A função que compõe este modelo tem como objetivo quantificar o tempo total de deslocamento $\left(T_{o}\right)$ de um veículo em uma rede, composta por uma série de $n_{c}$ cruzamentos, conforme a Equação (3.5).

$$
T_{o}=\sum_{i=1}^{n_{c}}\left(\frac{d}{V_{p}}+A_{v}\right)
$$

em que: $T_{o}$ : Tempo total de deslocamento de um veículo em uma rede semaforizada; $n_{c}$ : Número de cruzamentos; $d$ : comprimento da aproximação; $V_{p}$ : Velocidade esperada de deslocamento do fluxo na aproximação e $A_{v}$ : Atraso veicular.

O Modelo 2, semelhantemente ao Modelo 1, tem como variáveis os tempos de verde normal. Porém, no Modelo 2 busca-se calcular o tempo de verde ótimo $\left(g_{i}\right)$ da aproximação $i$ que possibilite minimizar o tempo total de deslocamento de um veículo em uma rede semaforizada. Assim, o Modelo 2 pode ser representado da seguinte forma:

$$
\begin{array}{cl}
\text { Minimizar } & T_{o}=\sum_{i=1}^{n_{c}}\left(\frac{d}{V_{p}}+A_{v}\right) \\
\text { Sujeito à } & T_{p} \leq g_{i} \leq C, i=1, \ldots, n_{c}
\end{array}
$$

em que: $T_{p}$ : Tempo perdido.

Como dados de entrada do problema tem-se: número de cruzamentos, fluxo de saturação e fluxo veicular de cada fase desses cruzamentos, tamanho do ciclo, tempo perdido, comprimento da aproximação e velocidade esperada da via. Observe-se que esses dois últimos grupos de dados não são necessários à aplicação do Modelo 1.

A partir da obtenção dos tempos de verde otimizados, as demais temporizações são obtidas de acordo com os diagramas de tempos disponível na literatura, em função do tamanho de ciclo atribuído (([2]), p. 62). 


\subsection{Implementação computacional dos modelos}

Os modelos propostos foram implementados em linguagem Fortran, tendo como base um programa desenvolvido por um dos autores ([6]) para a otimização com o emprego do método Simulated Annealing. A opção por este método deveu-se primordialmente a resultados satisfatórios anteriormente obtidos pelos autores a partir da aplicação deste a problemas de natureza combinatória, como pode ser visto, por exemplo, em [7] e [1].

O Simulated Annealing é um metodo de otimização classificado como uma meta-heurística, originado de um processo térmico, dito annealing ou recozimento, utilizado em metalurgia para obtenção de estados de baixa energia num sólido.

O método de otimização foi originalmente proposto por Kirkpatrick et al. ([5]), o qual se baseou na fundamentação teórica sobre a simulação do processo de recozimento proposto por Metropolis et al. ([11]). O Simulated Annealing tem como uma das principais vantagens permitir testar soluções distantes da solução atual, proporcionando um espaço de busca maior em relação aos métodos exatos. A técnica começa o processo de otimização a partir de uma solução inicial qualquer, considerada como solução atual, e para busca de valores otimizados são geradas soluções aleatórias para o problema. Se no processo surgirem soluções melhores, estas naturalmente são aceitas e o processo de busca reinicia a partir da nova solução encontrada. Porém, caso a solução gerada seja pior que a solução anterior, a aceitação será dada por um critério probabilístico, no qual se considera uma função de aceite, definida pela Equação (3.6).

$$
p=\exp \left(\frac{-\Delta f}{T}\right)
$$

em que: $T$ é um parâmetro do método, chamado de temperatura, que regula a probabilidade de pioras, e $\Delta f$ é a variação da função objetivo entre o valor da solução considerada como atual e a solução vizinha.

Calculada a função de aceite, um número aleatório é gerado no intervalo [0,1]. Se este número for menor ou igual a $p$, aceita-se a solução; em caso contrário, a solução atual é mantida. A temperatura é gradualmente reduzida, sendo o processo finalizado quando a temperatura assumir um valor pequeno ou o número máximo de iterações for atingido.

Para a execução dos programas são necessários os seguintes dados de entrada: fluxo de saturação e fluxo veicular de cada aproximação em cada cruzamento, tempo perdido e tamanho de ciclo. Para o Modelo 2, além dos dados citados, são necessários o fornecimento do comprimento da aproximação e a velocidade esperada em cada via.

Uma vez definida uma rede, a função objetivo é minimizada para o somatório das $n_{c}$ interseções dessa rede, tendo como variáveis de projeto os tempos de verde para programação dos semáforos. Um único tempo de verde de cada interseção é considerado como variável independente, visto que o tempo total do ciclo em cada cruzamento é predefinido. Assim, uma possível solução consiste num vetor formado por $n_{c}$ variáveis (tempo de verde). Cada variável pode assumir apenas valores inteiros (múltiplos de segundo), o que caracteriza o problema como de otimização 
combinatória. Os valores que podem ser assumidos pelas variáveis de projeto (restrições laterais) são determinados a partir dos valores de tamanho de ciclo e de tempo perdido fornecidos pelo usuário.

A qualidade de uma solução é medida pelo correspondente valor da função objetivo. Ressalta-se que o objetivo da otimização empregando o Modelo 1 consistiu em reduzir o atraso e o número de paradas sofridas por um veículo em uma aproximação, enquanto o Modelo 2 objetivou a minimização do tempo total de percurso veicular ao longo da rede.

Para o desenvolvimento do presente estudo foi empregado um microcomputador com processador Pentium de $2.1 \mathrm{GHz}, 3 \mathrm{~GB}$ de RAM e 250 GB de HDD. Para cada uma das análises efetuadas, o tempo de processamento máximo foi pouco inferior a 4 segundos.

\section{ESTUdO DA REGIÃO CENTRAL DE PASSO FUNDO}

\subsection{Descrição do problema}

Uma vez implementadas as formulações, diversas análises foram efetuadas para validação dos modelos. No presente item são apresentados alguns resultados obtidos. O estudo mais abrangente efetuado é descrito em Lacortt ([8]). Na sequência é apresentado um estudo em uma região central da cidade de Passo Fundo, RS, onde foram coletados dados reais referentes a esta região e, após, foram realizadas comparações entre os resultados das otimizações obtidos pelos modelos propostos e os dados reais praticados na região considerada. Além destas análises, também são apresentados os resultados obtidos de um estudo para verificar as influências que variações nos tempos de ciclo proporcionam aos índices de desempenho: atraso uniforme e grau de saturação.

Segundo Censo de 2010, a cidade de Passo Fundo possui área aproximada de 758,27 km², população aproximada de 184.869 habitantes e frota veicular, incluindo motocicletas, de 77.370. Tais características fazem de Passo Fundo uma cidade com alto fluxo veicular, onde nos últimos anos tornou-se rotineira a ocorrência de engarrafamentos, formações de filas e demais problemas consequentes dessa situação. Para a definição da rede selecionada para a otimização foi considerado o alto fluxo de veículos na região, sendo esta rede composta por cruzamentos semaforizados, definida pelos seguintes cruzamentos e vias: Av. Sete de Setembro com a Av. Brasil (daqui por diante designado como cruzamento 1), Rua Cel. Chicuta com a Av. Brasil (cruzamento 2), Av. Bento Gonçalves com a Av. Brasil (cruzamento 3) e Rua Fagundes dos Reis com a Av. Brasil (cruzamento 4).

Após a escolha da rede, a próxima etapa foi a obtenção dos dados de trânsito referentes à região escolhida, os quais serviram como parâmetros de entrada para a resolução computacional dos modelos, bem como os valores necessários para se determinarem as medidas de desempenho da rede.

Dessa forma, foi feito um levantamento de dados referentes ao trânsito da região selecionada. O fluxo de saturação $(S)$, o fluxo veicular $(q)$, os comprimentos das vias pertencentes aos cruzamentos e a temporização atual dos semáforos foram obtidos através de dados fornecidos pelo 
departamento de trânsito da Prefeitura Municipal de Passo Fundo. Quanto à velocidade esperada para via, esta foi definida pelos autores do presente trabalho, levando em conta as características de segurança e condições da trafegabilidade da via. Assim, para esta rede, as velocidades esperadas para as vias foi de $50 \mathrm{~km} / \mathrm{h}$ para a Av. Brasil e de $40 \mathrm{~km} / \mathrm{h}$ para as vias transversais. O tempo de amarelo considerado foi de 3 segundos, compatível com a recomendação da literatura técnica ([9]) para as velocidades consideradas.

O fluxo de saturação $(S)$ e fluxo veicular $(q)$, em veículos por hora de tempo verde, das vias pertencentes à rede estudada estão expostos na Tabela 1, onde a Fase 1 é composta pela Av. Brasil e, a Fase 2, pela vias transversais.

Tabela 1: Fluxo de saturação e fluxo veicular (em vht) nas vias da rede.

\begin{tabular}{|l|c|c|c|c|}
\hline & \multicolumn{2}{|c|}{ Fase 1} & \multicolumn{2}{c|}{ Fase 2} \\
\hline Cruzamentos & $S$ & $q$ & $S$ & $q$ \\
\hline 1. Av. Brasil × Av. Sete de Setembro & 2637 & 1397 & 3945 & 998 \\
\hline 2. Av. Brasil × Rua Cel. Chicuta & 2637 & 1201 & 3965 & 1352 \\
\hline 3. Av. Brasil × Av. Bento Gonçalves & 2550 & 1495 & 2820 & 642 \\
\hline 4. Av. Brasil $\times$ Rua Fagundes dos Reis & 2637 & 1655 & 4183 & 976 \\
\hline
\end{tabular}

Os comprimentos das vias pertencentes aos cruzamentos foram obtidos por meio de recursos do Google Maps ([10]), conforme ilustrado pela Tabela 2. Cada comprimento compreende a distância do cruzamento imediatamente anterior até o cruzamento em questão.

Tabela 2: Comprimento das vias, em metros, do percurso otimizado.

\begin{tabular}{|c|c|c|c|c|}
\hline & \multicolumn{4}{|c|}{ Cruzamentos } \\
\hline Ruas e Avenidas & 1 & 2 & 3 & 4 \\
\hline Av. Brasil & 62 & 100 & 260 & 280 \\
\hline Transversais & 130 & 150 & 130 & 140 \\
\hline
\end{tabular}

Para a obtenção da média de veículos retidos após o término do tempo de verde, é necessário obter a média entre a quantidade de veículos que chegam e param durante o tempo de vermelho (veículos em espera) e a quantidade de veículos que passam durante o tempo de verde. Assim, a quantidade de veículos retidos corresponde à diferença entre os dois.

$\mathrm{Na}$ busca da quantificação das medidas relatadas anteriormente, foi realizada uma pesquisa de campo, na qual foram coletadas dez amostras do número de veículos em espera durante o tempo de vermelho e do número de veículos que passam durante o tempo de verde, as quais foram obtidas pela contagem de veículos e foram realizadas em dois dias, nos intervalos entre 11 e $12 \mathrm{~h}$ e entre 18 e 19h. A primeira foi coletada em uma sexta-feira, dia 8 de abril de 2011, e a outra ocorreu em uma segunda-feira, dia 11 de abril de 2011. Justifica-se a escolha desses horários 
por serem os de maior fluxo veicular, segundo informações de técnicos da Prefeitura Municipal de Passo Fundo. Com os valores obtidos com as dez amostras em ambos os horários e dias, obtiveram-se os valores médios de veículos em espera e de veículos que passam a aproximação.

\subsection{Resultados numéricos}

Com o objetivo de obterem-se novos planos semafóricos otimizados, foi efetuada a resolução do estudo de caso da região central de Passo Fundo por meio dos dois modelos apresentados.

Em relação ao Modelo 1 foram realizadas otimizações com variações do fator de penalidade de parada $(K)$, de 10 em 10 unidades, em um intervalo de 10 a 60. Neste caso, os tempos permaneceram os mesmos com os diferentes valores de $K$. Após, foi realizada a otimização com o Modelo 2, para o qual foram obtidos os mesmos tempos do Modelo 1 para o caso analisado. Assim, os resultados listados na sequência do presente trabalho referem-se a ambos os modelos estudados.

As temporizações antes da otimização (atuais) e após a otimização estão expostas nas Tabelas 3 e 4. Na Tabela 3, cada tempo e vias são definidos pelas seguintes siglas: $T_{p}$ é o tempo perdido; $V e r$ é o tempo de vermelho; $g$ é o tempo de verde; $C$ é o tempo de ciclo; $A v$ é a Avenida Brasil e tran, as demais vias transversais.

Tabela 3: Tempos semafóricos atuais e otimizados.

\begin{tabular}{|c|c|c|c|c|c|c|}
\hline \multicolumn{7}{|c|}{ Atuais } \\
\hline Cruzamentos & $T_{p}$ & $g_{a v}$ & Ver $_{a v}$ & $g_{\text {tran }}$ & Ver $_{\text {tran }}$ & $C$ \\
\hline 1 & 19 & 40 & 28 & 25 & 43 & 84 \\
\hline 2 & 19 & 40 & 28 & 25 & 43 & 84 \\
\hline 3 & 19 & 40 & 28 & 25 & 43 & 84 \\
\hline 4 & 19 & 40 & 28 & 25 & 43 & 84 \\
\hline Otimizados \\
\hline Cruzamentos & $T_{p}$ & $g_{a v}$ & $V^{2} r_{a v}$ & $g_{\text {tran }}$ & Ver tran & $C$ \\
\hline 1 & 19 & 44 & 24 & 21 & 48 & 84 \\
\hline 2 & 19 & 37 & 31 & 28 & 41 & 84 \\
\hline 3 & 19 & 46 & 22 & 19 & 50 & 84 \\
\hline 4 & 19 & 45 & 23 & 20 & 49 & 84 \\
\hline
\end{tabular}

Visando comparar se ocorreriam melhoras com a implementação dos tempos sugeridos pelos modelos em relação aos tempos atuais, também foi realizada uma análise dos indicativos de desempenho da região após a otimização, caso esses tempos fossem implementados. Os indicativos de desempenho definidos para esta análise foram o grau de saturação, o atraso uniforme e o número de veículos retidos após o tempo de verde. O indicativo de desempenho grau de saturação antes e após a otimização estão apresentados na Tabela 4. 
Tabela 4: Graus de saturação $(X)$, com tempos semafóricos atuais e otimizados.

\begin{tabular}{|c|c|c|c|c|c|c|c|c|}
\hline Cruzamentos & \multicolumn{2}{|c|}{1} & \multicolumn{2}{c|}{2} & \multicolumn{2}{c|}{3} & \multicolumn{2}{c|}{4} \\
\hline Aprox. & Brasil & Sete & Brasil & Chicuta & Brasil & Bento & Brasil & F. Reis \\
\hline Atuais & 1,11 & 0,85 & 0,95 & 1,15 & 1,23 & 0,76 & 1,32 & 0,78 \\
\hline Otimizados & 1,01 & 1,01 & 1,03 & 1,02 & 1,07 & 1,01 & 1,17 & 0,98 \\
\hline
\end{tabular}

Diante dos resultados, observa-se que ocorreriam melhoras no grau de saturação nas vias, principalmente nos superiores a 1, tendo em vista que o ideal é o grau de saturação ser inferior a 1. Porém, para o tamanho de ciclo e de tempo perdido atuais, isso não foi possível. Outra vantagem que pode ser observada nos valores do grau de saturação correspondentes à solução otimizada é que tendem a ficar equilibrados entre as fases de cada cruzamento, proporcionando, assim, igualdade de condições de trafegabilidade em ambas as direções.

$\mathrm{Na}$ busca de verificar as influências que as mudanças dos tempos de ciclo proporcionam nas temporizações semafóricas e nos índices de desempenho atraso uniforme e grau de saturação, a rede do exemplo foi otimizada pelo Modelo 1, com os tempos de ciclo em um intervalo de 84 a $114 \mathrm{~s}$, variando de $10 \mathrm{em} \mathrm{10s,} \mathrm{obtendo} \mathrm{os} \mathrm{resultados} \mathrm{da} \mathrm{Tabela} 5$.

Tabela 5: Tempos, graus de saturação e atraso uniforme para diferentes ciclos.

\begin{tabular}{|c|c|c|c|c|c|c|c|}
\hline Ciclo & Cruzamentos & $T_{p}$ & $g_{a v}$ & $X_{a v}$ & $g_{\text {tran }}$ & $X_{\text {tran }}$ & $A U$ \\
\hline 84 & 1 & 19 & 44 & 1,01 & 21 & 1,01 & 51,88 \\
\hline 84 & 2 & 19 & 37 & 1,03 & 28 & 1,02 & 52,47 \\
\hline 84 & 3 & 19 & 46 & 1,07 & 19 & 1,01 & 53,34 \\
\hline 84 & 4 & 19 & 45 & 1,17 & 20 & 1,00 & 56,11 \\
\hline \hline 94 & 1 & 19 & 51 & 0,98 & 24 & 0,99 & 55,81 \\
\hline 94 & 2 & 19 & 43 & 1,00 & 32 & 1,00 & 56,43 \\
\hline 94 & 3 & 19 & 54 & 1,02 & 21 & 1,01 & 57,27 \\
\hline 94 & 4 & 19 & 53 & 1,11 & 22 & 1,00 & 59,97 \\
\hline \hline 104 & 1 & 19 & 57 & 0,97 & 28 & 0,94 & 59,75 \\
\hline 104 & 2 & 19 & 49 & 0,97 & 36 & 0,99 & 60,44 \\
\hline 104 & 3 & 19 & 61 & 1,00 & 24 & 0,99 & 61,32 \\
\hline 104 & 4 & 19 & 61 & 1,07 & 24 & 1,00 & 64,00 \\
\hline \hline 114 & 1 & 19 & 64 & 0,94 & 31 & 0,93 & 63,77 \\
\hline 114 & 2 & 19 & 54 & 0,96 & 41 & 0,95 & 64,46 \\
\hline 114 & 3 & 19 & 68 & 0,98 & 27 & 0,96 & 65,41 \\
\hline 114 & 4 & 19 & 68 & 1,05 & 27 & 0,99 & 68,22 \\
\hline
\end{tabular}


Observou-se que o aumento do ciclo propicia uma redução no grau de saturação, proporcionando, dessa forma,melhoras para este indicativo. No entanto, mesmo para o maior tempo de ciclo analisado (de 30 segundos acima do atualmente praticado), o grau de saturação ainda estaria situado acima dos índices recomendados, uma vez que um índice superior a 1 indica que nem todos os veículos que chegam ao cruzamento seriam atendidos.

Em contraposição, o aumento do tempo de ciclo acarreta um maior tempo de espera durante o tempo de vermelho, influenciando diretamente no aumento do atraso uniforme. Assim, na escolha pelo melhor tempo de ciclo neste caso, teria de ser levado em conta o que proporciona melhor qualidade de trafegabilidade ao usuário da via: o esvaziamento total da via (caixa) ao término do sinal verde (grau de saturação) ou a redução do tempo de espera no sinal vermelho (atraso uniforme).

Entende-se que, no caso, uma alternativa viável, e pensando estritamente na melhoria do fluxo veicular, seria a redução do tempo perdido, atualmente em 19 segundos. Esse tempo é excepcionalmente alto pois inclui um período de 10 segundos de vermelho total, durante o qual todos os semáforos do cruzamento estão fechados.

\section{CONCLUSÕES}

O presente estudo objetivou a aplicação de técnica de otimização para a minimização do tempo de percurso veicular em vias semaforizadas. Assim, dois modelos foram estudados e implementados, tendo o tempo de verde de cada semáforo como variável de projeto. Diante dos resultados obtidos com o estudo, se observa que os tempos otimizados pelos dois modelos foram os mesmos, sendo que estes, em comparação com os tempos atuais, apresentaram melhoras significativas nos indices de desempenho das vias, reduzindo o número de veículos retidos após o término do tempo de verde, bem como o grau de saturação e o atraso uniforme.

Já com relação às mudanças nas temporizações do tamanho de ciclo, observam-se melhorias no grau de saturação com o aumento do ciclo, conduzindo em contrapartida ao aumento do atraso uniforme. Considerando que, mesmo um aumento significativo no tamanho do ciclo não possibilita o total esvaziamento da via ao término do sinal verde, sugere-se a redução no tempo perdido como uma forma de reduzir o ciclo ótimo e, consequentemente, o grau de saturação e o atraso uniforme. Os resultados obtidos sugerem que a adoção, em cidades de médio e grande porte, da metodologia proposta no presente trabalho, pode proporcionar um melhor desempenho no fluxo veicular. No caso específico da região central da cidade de Passo Fundo, observou-se uma melhora significativa nos índices de desempenho das vias estudadas, consistindo unicamente num melhor aproveitamento dos recursos já existentes e implantados.

Cabe enfatizar que o sucesso na aplicação dos modelos está diretamente relacionado à sincronização dos semáforos componentes da rede, a qual, neste estudo, não foi diretamente abordada.

\footnotetext{
ABSTRACT. This work presents two mathematical optimization models, developed in order to minimize the course time delay in cities of medium and large size and taking the time of green in each traffic signal as the design variables. Some computational results are also
} 
presented, based on data collected for the transit of a central region of the city of Passo Fundo, RS. The results obtained point to the application of these formulations to real situations.

Keywords: transit, combinatorial optimization, semaphore programming.

\section{REFERÊNCIAS}

[1] F.R. Barao, M. Kripka \& R.M.L. Kripka. Modelagem Matematica para Coleta Seletiva no Municipio de Passo Fundo-RS, "Meta-heuristicas em Pesquisa Operacional" (H.S. Lopes and L.C.A. Rodrigues and M.T.A. Steiner, eds.), pp. 237-252, Omnipax, Curitiba, (2013).

[2] Brasil. Manual de semáforos, 2. Ed, Brasília, Departamento Nacional de Trânsito (DENATRAN), (1984).

[3] Chile. Manual de señalización de tránsito. Disponível em:

$<$ http://www.subtrans.cl/subtrans/documentos/senalizacion.html>. Acesso em: 1 out, 2011.

[4] A.F. Gobbo. "Proposta de aplicação do sistema de inferência Neuro-Fuzzi pra otimização de tráfego", Dissertação Mestrado, CEFET, Curitiba, PR, (2005).

[5] S. Kirkpatrick, C.D. Gelatt \& M.P. Vecchi. Optimization by simulated annealing. Science, 220 (4598) (1983), 671-680.

[6] M. Kripka. Discrete optimization of trusses by simulated annealing. Journal of the brazilian society of mechanical sciences and engineering, 26(2) (2004), 170-173.

[7] R.M.L. Kripka, M. Kripka \& M.C. Silva. Formulação para o problema de alocação de salas de aula com minimização de deslocamentos. "XLIII Simpósio Brasileiro de Pesquisa Operacional”, Ubatuba, SP. Anais do XLIII SBPO, (2011).

[8] M. Lacortt. "Modelagem matemática para otimização do tráfego urbano semaforizado", Dissertação Mestrado, Universidade de Passo Fundo - UPF, Passo Fundo, RS, (2011).

[9] C.M.V. Lima. "Otimização de trânsito: Uma abordagem utilizando algoritmos genéticos", 2005. Disponível em: <http://www.cin.ufpe.br/ tg/2005-1/cmvl.doc>. Acesso em: 20 maio 2011.

[10] MAPS. "Mapa da cidade de Passo Fundo". Google MAPS. Disponível em: $<$ http://maps.google.com.br/>. Acesso em: 20 out 2011.

[11] N. Metropolis et al. Equation of State Calculations by Fast Computing Machines. The Journal of Chemical Physics, 21(6) (1953), 1087-1092.

[12] J.C. Piai \& S.G. de Cervantes. Um modelo para tráfego urbano e suas otimizações, "XVIII Congresso Brasileiro de Automática", Bonito-MS. Anais..., (2010).

[13] D.I. Robertson. Research on Transyt ans Scoot methods of signal coordination. ITE Journal, em (CD ROM) ITE Digital Library, 1997, (1986), 36-40.

[14] L. Vilanova. "SIRI: Um novo simulador para redes de semáforo", 2005. Disponível em: $<$ http//meusite.mackenzie.com.br/professor_cucci/texto29.pdf>. Acesso em: 10 mar 2011.

[15] P.C.M. Silva. "Elementos do sistema de tráfego", 2001. Disponível em: <http://www.unb.br/ft/enc/pagdisc/engtraf/apostilas/APOSTILA1.pdf>. Acesso em: 4 jun 2011. 